\title{
Gender differences in taste and foods habits
}

Mauro Lombardo, Giovanni Aulisa and Elvira Padua

Department of Human Sciences and Promotion of the Quality of Life,

San Raffaele Roma Open University, Rome, Italy

Giuseppe Annino

Department of Medicine Systems, University of Rome "Tor Vergata", Rome, Italy and Department of Human Sciences and Promotion of the Quality of Life,

San Raffaele Roma Open University, Rome, Italy

Ferdinando Iellamo

Department of Clinical Sciences and Translational Medicine,

University of Tor Vergata, Rome, Italy

Antonio Pratesi

Azienda ULSS n 2 Marca Trevigiana, Treviso, Italy

Massimiliano Caprio

Laboratory of Cardiovascular Endocrinology, IRCCS San Raffaele Pisana, Rome, Italy and Department of Human Sciences and Promotion of the Quality of Life, San Raffaele Roma Open University, Rome, Italy, and

\section{Alfonso Bellia}

Department of Systems Medicine, University of Rome "Tor Vergata", Rome, Italy and Department of Human Sciences and Promotion of the Quality of Life, San Raffaele Roma Open University, Rome, Italy

\begin{abstract}
Purpose - The purpose of this paper is to examine gender differences in food habits and food choices, including decisions in healthy eating, to personalize diet therapies to be as effective possible for long-term weight loss.

Design/methodology/approach - In this cross-sectional study, eating behaviours were assessed using a questionnaire composed of 12 questions concerning food habits, 17 concerning food taste, and four about healthy eating. There were 2,021 (1,276 women) Caucasian adults enrolled in the study.

Findings - Statistically significant differences in women compared to men occurred for the following questionnaire entries reading eating habits: whole grain food (10.0 per cent higher in women; $p<0.001)$; cereals such as barley (8.3 per cent higher in women, $p<0.001)$; cooked vegetables (6.6 per cent higher in women, $p<0.001$ ); eggs (5.0 per cent lower in women, $p=0.03)$; meat (9.3 per cent lower in women, $p<0.001)$; and processed meat (7.1 per cent lower in women, $p<0.001$ ). Women consume more water, sugar-sweetened beverages and alcoholic drinks than males, and liked salty foods more than sweet foods. Men ate faster, ate more during the night and slept worse than women. Men ate meals out more often and tended to be hungrier later in the day. Women missed more meals and ate more times during the day and were also more likely to eat uncontrollably.
\end{abstract}

Conflict of interest: The authors declare that they have no conflict of interest. This research received no specific grant from any funding agency in the public, commercial or not-for-profit sectors.
Received 17 April 2019 Revised 7 June 2019 Accepted 7 June 2019 
Research limitations/implications - The authors observed strong evidence of profound genderspecific differences between men and women in terms of dietary habits, the taste of food and in the relationship with meals.

Practical implications - The findings suggest a need for the creation of gender-specific programs for promoting a healthy lifestyle.

Social implications - A need for the creation of gender-related programs for promoting healthy lifestyle has been demonstrated.

Originality/value - Reasons for the different eating behaviours among men and women have been found. Western society's perception of the ideal body weight is much lower for women than for men. In general, social perceptions influence nutritional behaviour to a great extent. Women's greater nutritional knowledge and sex-specific taste preferences also account for the differences in eating behaviour.

Keywords Diet, Weight loss, Mediterranean diet, Eating habits, Gender differences, Healthy eating behaviour

Paper type Research paper

\section{Introduction}

The most suitable diet for human beings and the well-being of the planet has been well characterized since the 1950s. A diet focused on vegetables, whole grains, fruit, legumes, oilseeds, small blue fish and yoghurt can potentially prevent and cure most chronic degenerative diseases of the modern era (Franquesa et al., 2019). The same nutritional model is probably the best choice to help save the Earth (Springmann et al., 2018). However, the effectiveness of long-term nutritional therapies is very low (Yumuk et al., 2015). A multidisciplinary approach, a personalized lifestyle change (MacLean et al., 2014) and diets that do not involve high amounts of caloric restriction (Lombardo et al., 2017) are the therapies that have proven to be the most effective in the long term.

New diagnostic tools are needed to gain an in-depth understanding of subjects' lifestyle habits and to prepare the most effective nutritional therapies. One of the most interesting aspects of this is the understanding of gender differences in relationships with food. Diets with the same amount of calorie restriction are often less effective for women than for men (Williams et al., 2014).

Several studies have demonstrated important gender differences in relationships with food. Beardsworth et al. showed that women have more "virtuous" food choice patterns in terms of improving health but more food-related guilt and "disordered" eating than men (Beardsworth et al., 2002). Gender differences were also observed for specific food categories (Padulo et al., 2017), probably due to differing mechanisms in the superior temporal sulcus (STS) that account for food preference (Manippa et al., 2017). Gender-specific differences in nutritional knowledge become obvious during adolescence and persist into adulthood (Davy et al., 2006). For instance, women tend to characterize meat and meat-eating experiences negatively (Kubberød et al., 2002). Men often have less nutritional knowledge, and their approach to food is more frequently uncomplicated and more pleasure oriented than women. Females instead preferred comfort foods that were more snack related (such as chocolate and ice cream) (Wansink et al., 2003) and more frequently present with very serious health complications as a result of eating disorders (Di Cola et al., 2014). For men, sports and exercise are more important than nutrition to their health and beauty (Kiefer et al., 2005).

To our knowledge, few studies have evaluated food gender preferences and healthy eating in adults in a large sample of subjects who want to improve their lifestyle. 


\section{Methods}

Subjects

The final sample was comprised of 2,021 subjects (1,276 females and 745 males). They were enrolled in a weight-loss intervention at an obesity centre in Rome, Italy. Exclusion criteria were as follows: age $<12$ years or $>75$ years; pregnancy or nursing; body mass index (BMI) $<$ $18.5 \mathrm{~kg} \cdot \mathrm{m}^{-2}$ or $>40 \mathrm{~kg} \cdot \mathrm{m}^{-2}$ and any lifestyle treatment in the year before the study. All subjects underwent an online test survey before the visit and gave their own written informed consent to participate. Data collection was performed between June 2015 and January 2019. Online support was provided. Study protocols including consent form has been approved by IRCCS San Raffaele ethical body. All procedures performed were in accordance with the ethical standards of the 1964 Helsinki declaration and its later amendments.

\section{Study procedures and diagnostic criteria}

The online questionnaire was structured and self-administered. It consisted of three parts. In agreement with others, the instrument incorporated food habits, taste and possible eating disorder questions that were important for dietary gender differences. We did not conduct a formal test of validity, but the questionnaire was similar to other validated food taste questionnaires (Carbonneau et al., 2017).

The first part queried how many times subjects ate per day and when they were hungry. They were also questioned whether they usually miss meals, ever eat distracted or not at the table or eat fast. The differences between normal days and weekends were evaluated by questions about eating out for meals and if they ate differently on the weekends. Eating disorders were also investigated by questions about uncontrollable eating (even if not hungry) or waking up at night to eat. To explore the relationship between hunger and sleep deprivation, how the subject slept at night and if they slept bad because of struggling to fall asleep, woke up a lot sooner than they would have liked or woke up several times during the night was queried.

In the second part, the question "Do you like the following foods?" queried subjects about tastes of each food group individually ("like”, "don't like" and "don't know"). Subjects selfreported their nutritional tastes in a food frequency questionnaire comprising cow's milk, vegetable drinks (e.g. soy milk), low-fat and low-sugar yogurt, fresh cheeses, meat, processed meat (e.g. prosciutto), fish, eggs, legumes, cooked vegetables, raw vegetables, fruits, cereals (e.g. spelt and barley), whole grains, nuts, tofu and dark chocolate (cocoa $>75$ per cent).

The third part of the questionnaire asked about how many litres of water were drank per day, how many times alcoholic beverages were consumed in a week and how many sugarsweetened beverages were consumed per day.

\section{Statistical analysis}

Statistical analysis was performed using the SOFA STATISTICS (software version 1.4.6, Paton-Simpson and Associates, Auckland, New Zealand). Descriptive statistics (frequencies) described the taste and food consumption patterns of the subjects (for the whole sample and also separately for men and women). Chi-squared statistics were used to test the overall differences between male and female individuals (statistical significance set at $p \leq 0.05$ ).

\section{Results}

\section{Food taste preferences}

As Table I shows women preferred more whole grain foods (83.5 vs 73.6 per cent; $p<0.001$ ), cereals (e.g. spelt and barley) ( 80.5 vs 72.2 per cent; $p<0.001$ ) and cooked vegetables (93.0 vs 86.4 per cent; $p<0.001$ ) than men. Men seemed to like more eggs (82.9 vs 87.9 per cent; 
$p=0.03$ ), meat (82.1 vs 91.4 per cent; $p<0.001$ ) and processed meat (e.g. prosciutto) (83.4 vs 90.5 per cent; $p<0.001$ ) than women. Answers of "rarely" to food tastes were not considered.

Other differences between men and women regarding tastes were not significant (ns), including cow's milk (59.4 vs 60.8 per cent; $p=n s$ ), low-fat and low-sugar yogurt (54.8 vs 58.3 per cent; $p=\mathrm{ns}$ ), vegetable drinks (e.g. soy milk) (38.2 vs 33.4 per cent; $p=\mathrm{ns}$ ), raw vegetables ( $51.8 \mathrm{vs}$ 45.9 per cent; $p=\mathrm{ns}$ ), fruits (90.7 vs 89.9 per cent; $p=\mathrm{ns}$ ), legumes (85.3 vs 87 per cent; $p=\mathrm{ns}$ ), fish (81.8 vs 86.0 per cent; $p=n$ ), fresh cheeses ( 82.8 vs 80.7 per cent; $p=n s$ ), nuts ( 85.6 vs 81.5 per cent; $p=\mathrm{ns})$, tofu ( 23.4 vs 20.4 per cent; $p=\mathrm{ns}$ ) and dark chocolate (cocoa $>75.0$ per cent) (74.7 vs 74.8 per cent; $p=$ ns) (Table II).

\section{Water, sugar-sweetened beverages and alcohol preferences}

On average, women drank less water ( $\leq 11$ per day 55.1 per cent vs 40.3 per cent; $p<0.001$ ), fewer sugar-sweetened beverages ( 0 drinks per day: 35.4 per cent vs 26.6 per cent; $p<0.001$ ) and fewer alcoholic drinks ( 0 drinks per day 41.8 vs 30.1 per cent; $p<0.001)$ than men in a day. Women also preferred salty foods more than sweet foods compared to men $(p<0.001)$ (Table III).

\section{Meal behaviour}

Men ate faster 67.5 vs 79.3 per cent; $p<0.001$ ), while women missed more meals (26.9 vs 21.3 per cent; $p<0.001$ ). Men skipped breakfast more often (10 vs 17 per cent; $p<0.001$ ). Women ate uncontrollably, even if not hungry, more frequently (80.6 vs 69 per cent; $p<0.001$ ), while more men woke up at night to eat (19.8 vs 28.2 per cent; $p<0.001)$. Women seemed to be more hungry in the first part of the day than the second $(p=0.024)$, while men seemed to eat less times per day $(p<0.001)$. Men usually ate out from home more often 67.8 vs 75.5 per cent; $p<0.001$ ), ate more often in a refectory setting at lunch and more often ate foods from home at work than women; women usually ate together in restaurants more often while on lunch breaks from work (Table IV).

No significant gender differences were seen when asked about eating differently on the weekends compared to working days (79.1 vs 76.9 per cent; $p=n s$ ), eating alone (49.2 vs 42 per cent; $p=\mathrm{ns}$ ) and eating while distracted by smartphones or televisions or not at the table (66.1 vs 62.5 per cent; $p=\mathrm{ns}$ ).

\section{Sleep habits}

Women more frequently slept better than men (50.9 vs 45.4 per cent; $p<0.001$ ), but women reported waking up several times during the night (9.38 per cent more than men; $p<0.001$ ) (Table III).

All data not shown have been included in the additional material. Data set is available at Mendeley Data, v1 http://dx.doi.org/10.17632/cy8zywfhr3.1

Table I.

Differences between women and men in food preferences

\begin{tabular}{lcc}
\hline Type of food & Difference $\%$ woman vs men & $p$ \\
\hline Whole grains food & 10 & $<0.001$ \\
Cereals & 8 & $<0.001$ \\
Cooked vegetables & 6,6 & $<0.001$ \\
Eggs & -5 & 0.03 \\
Meat & $-9,3$ & $<0.001$ \\
Processed meat & $-7,1$ & $<0.001$ \\
\hline
\end{tabular}




\begin{tabular}{|c|c|c|c|c|c|c|c|c|c|c|}
\hline \multirow[b]{2}{*}{ Question } & \multirow[b]{2}{*}{ Answer } & \multicolumn{2}{|c|}{$\begin{array}{c}\text { Total (2021 } \\
\text { subjects) }\end{array}$} & \multicolumn{2}{|c|}{$\begin{array}{l}\text { Women } \\
\text { (1276 } \\
\text { subjects) }\end{array}$} & \multicolumn{2}{|c|}{$\begin{array}{l}\text { Men (745 } \\
\text { subjects) }\end{array}$} & \multirow{2}{*}{$\begin{array}{c}\text { Difference } \\
{[\%]}\end{array}$} & \multirow[b]{2}{*}{$p$} & \multirow[t]{2}{*}{$\begin{array}{l}\text { Taste and } \\
\text { foods habits }\end{array}$} \\
\hline & & No. & {$[\%]$} & No. & {$[\%]$} & No. & {$[\%]$} & & & \\
\hline $\begin{array}{l}\text { Do you eat whole } \\
\text { grains food? }\end{array}$ & $\begin{array}{l}\text { Don't know } \\
\text { NO } \\
\text { Rarely } \\
\text { Yes }\end{array}$ & $\begin{array}{r}16 \\
114 \\
277 \\
1614\end{array}$ & $\begin{array}{r}0.8 \\
5.6 \\
13.7 \\
79.9\end{array}$ & $\begin{array}{r}5 \\
63 \\
142 \\
1066\end{array}$ & $\begin{array}{r}0.4 \\
4.9 \\
11.1 \\
83.5\end{array}$ & $\begin{array}{r}11 \\
51 \\
135 \\
548\end{array}$ & $\begin{array}{c}1.5 \\
6.85 \\
18.1 \\
73.56\end{array}$ & $\begin{array}{l}-1.1 \\
-1.9 \\
-7.0 \\
10\end{array}$ & $<0.001$ & \\
\hline $\begin{array}{l}\text { Do you eat cereals } \\
\text { (e.g. spelled, barley) }\end{array}$ & $\begin{array}{l}\text { Don't know } \\
\text { NO } \\
\text { Rarely } \\
\text { Yes }\end{array}$ & $\begin{array}{r}20 \\
173 \\
263 \\
1565\end{array}$ & $\begin{array}{r}1.0 \\
8.6 \\
13.0 \\
77.4\end{array}$ & $\begin{array}{r}8 \\
111 \\
130 \\
1027\end{array}$ & $\begin{array}{r}0.6 \\
8.7 \\
10.2 \\
80.5\end{array}$ & $\begin{array}{r}12 \\
62 \\
133 \\
538\end{array}$ & $\begin{array}{r}1.6 \\
8.3 \\
17.8 \\
72.2\end{array}$ & $\begin{array}{r}-1.0 \\
0.4 \\
-7.7 \\
8.3\end{array}$ & $<0.001$ & \\
\hline $\begin{array}{l}\text { Do you eat cooked } \\
\text { vegetables? }\end{array}$ & $\begin{array}{l}\text { Don't know } \\
\text { NO } \\
\text { Rarely } \\
\text { Yes }\end{array}$ & $\begin{array}{r}6 \\
50 \\
134 \\
1831\end{array}$ & $\begin{array}{r}0.3 \\
2.5 \\
6.6 \\
90.6\end{array}$ & $\begin{array}{r}3 \\
25 \\
61 \\
1187\end{array}$ & $\begin{array}{r}0.2 \\
2.0 \\
4.8 \\
93.0\end{array}$ & $\begin{array}{r}3 \\
25 \\
73 \\
644\end{array}$ & $\begin{array}{r}0.4 \\
3.4 \\
9.8 \\
86.4\end{array}$ & $\begin{array}{r}-0.2 \\
-1.4 \\
-5.0 \\
6.6\end{array}$ & $<0.001$ & \\
\hline Do you eat eggs? & $\begin{array}{l}\text { Don't know } \\
\text { NO } \\
\text { Rarely } \\
\text { Yes }\end{array}$ & $\begin{array}{r}3 \\
89 \\
216 \\
1713\end{array}$ & $\begin{array}{r}0.2 \\
4.4 \\
10.7 \\
84.8\end{array}$ & $\begin{array}{r}2 \\
63 \\
153 \\
1058\end{array}$ & $\begin{array}{l}0.2 \\
4.9 \\
12 \\
82.9\end{array}$ & $\begin{array}{r}1 \\
26 \\
63 \\
655\end{array}$ & $\begin{array}{r}0.1 \\
3.5 \\
8.5 \\
87.9\end{array}$ & $\begin{array}{l}0 \\
1.4 \\
3.5 \\
-5\end{array}$ & $p=0.03$ & \\
\hline Do you eat meat? & $\begin{array}{l}\text { Don't know } \\
\text { NO } \\
\text { Rarely } \\
\text { Yes }\end{array}$ & $\begin{array}{r}9 \\
113 \\
170 \\
1729\end{array}$ & $\begin{array}{r}0.4 \\
5.6 \\
8.4 \\
85.6\end{array}$ & $\begin{array}{r}6 \\
90 \\
132 \\
1048\end{array}$ & $\begin{array}{c}0.5 \\
7 \\
10.3 \\
82.1\end{array}$ & $\begin{array}{r}3 \\
23 \\
38 \\
681\end{array}$ & $\begin{array}{r}0.4 \\
3.1 \\
5.1 \\
91.4\end{array}$ & $\begin{array}{l}0.1 \\
4 \\
5.2 \\
-9.3\end{array}$ & $<0.001$ & \\
\hline $\begin{array}{l}\text { Do you eat processed } \\
\text { meat (es prosciutto)? }\end{array}$ & $\begin{array}{l}\text { Don't know } \\
\text { NO } \\
\text { Rarely } \\
\text { Yes }\end{array}$ & $\begin{array}{r}7 \\
161 \\
115 \\
1738\end{array}$ & $\begin{array}{l}0.3 \\
8 \\
5.7 \\
86\end{array}$ & $\begin{array}{r}3 \\
126 \\
83 \\
1064\end{array}$ & $\begin{array}{r}0.2 \\
9.9 \\
6.5 \\
83.4\end{array}$ & $\begin{array}{r}4 \\
35 \\
32 \\
674\end{array}$ & $\begin{array}{r}0.5 \\
4.7 \\
4.3 \\
90.5\end{array}$ & $\begin{array}{r}-0.3 \\
5.2 \\
2.2 \\
-7.1\end{array}$ & $<0.001$ & $\begin{array}{r}\text { Table II } \\
\text { Food taste } \\
\text { differences between } \\
\text { women and men }\end{array}$ \\
\hline
\end{tabular}

\section{Discussion}

Explanations for the different eating behaviours among men and women can be found in psychological and sociocultural factors.

In the Western society, the ideal body weight is perceived to be much lower for women than for men. In general, social perceptions influence nutritional behaviour to a great extent. Our data showed that women more often than men have an ambivalent relationship with food. In fact, women usually give greater importance to healthy eating while orienting themselves more strongly with social norms. Starting from adolescence, women are reported to have a higher intake of fruits and vegetables, a higher intake of dietary fibre and a lower intake of fat (Djordjevic-Nikic and Dopsaj, 2013). Another factor that might contribute to gender differences in diet is the greater concern of women about weight control, and their higher frequency of dieting (Wardle et al., 2004). The greater nutritional knowledge of women and sex-specific taste preferences also account for the differences in eating behaviour (Baker and Wardle, 2003).

We discovered that women eat uncontrollably (even if not hungry) and miss meals more frequently than men. Young females usually pay more attention to diet and snack less than young males. As they grow older, food repertoire widens due to social and cognitive influences ( $\mathrm{Nu}$ et al., 1996). These gender differences may be due to the hormonal regulation 
NFS

Table III.

Differences in food habits between women and men

\begin{tabular}{|c|c|c|c|c|c|c|c|c|c|}
\hline \multirow[b]{2}{*}{ Questions } & \multirow[b]{2}{*}{ Answers } & \multicolumn{2}{|c|}{$\begin{array}{c}\text { Total (2021 } \\
\text { subjects) }\end{array}$} & \multicolumn{2}{|c|}{$\begin{array}{l}\text { Women (1276 } \\
\text { subjects) }\end{array}$} & \multicolumn{2}{|c|}{$\begin{array}{l}\text { Men }(745 \\
\text { subjects) }\end{array}$} & \multirow{2}{*}{$\begin{array}{c}\text { Difference } \\
{[\%]}\end{array}$} & \multirow[b]{2}{*}{$p$} \\
\hline & & No. & {$[\%]$} & No. & {$[\%]$} & No. & {$[\%]$} & & \\
\hline $\begin{array}{l}\text { How many litres of water } \\
\text { do you drink per day on } \\
\text { average? }\end{array}$ & $\begin{array}{l}0.0 \\
1.0 \\
2.0 \\
3.0 \\
4.0 \\
5.0\end{array}$ & $\begin{array}{r}29 \\
1003 \\
803 \\
161 \\
20 \\
5\end{array}$ & $\begin{array}{r}1.4 \\
49.6 \\
39.7 \\
8.0 \\
1.0 \\
0.2\end{array}$ & $\begin{array}{r}23 \\
703 \\
469 \\
73 \\
8 \\
0\end{array}$ & $\begin{array}{r}1.8 \\
55.1 \\
36.8 \\
5.7 \\
0.6 \\
0.1\end{array}$ & $\begin{array}{r}6 \\
300 \\
334 \\
88 \\
12 \\
5\end{array}$ & $\begin{array}{r}0.8 \\
40.3 \\
44.8 \\
11.8 \\
1.6 \\
0.7\end{array}$ & $\begin{array}{r}1.0 \\
14.8 \\
-8.1 \\
-6.1 \\
-1.0 \\
-0.7\end{array}$ & $<0.001$ \\
\hline $\begin{array}{l}\text { How many sugary drinks } \\
\text { or added sugar do you } \\
\text { consume per day on } \\
\text { average? }\end{array}$ & $\begin{array}{l}0.0 \\
1.0 \\
2.0 \\
3.0 \\
4.0 \\
5.0 \\
6.0 \\
7.0\end{array}$ & $\begin{array}{r}650 \\
433 \\
339 \\
295 \\
170 \\
58 \\
23 \\
53\end{array}$ & $\begin{array}{r}32.2 \\
21.4 \\
16.8 \\
14.6 \\
8.4 \\
2.9 \\
1.1 \\
2.6\end{array}$ & $\begin{array}{r}452 \\
267 \\
225 \\
157 \\
96 \\
34 \\
12 \\
33\end{array}$ & $\begin{array}{r}35.4 \\
20.9 \\
17.6 \\
12.3 \\
7.5 \\
2.7 \\
0.9 \\
2.6\end{array}$ & $\begin{array}{r}198 \\
166 \\
114 \\
138 \\
74 \\
24 \\
11 \\
20\end{array}$ & $\begin{array}{r}26.6 \\
22.3 \\
15.3 \\
18.5 \\
9.9 \\
3.2 \\
1.5 \\
2.7\end{array}$ & $\begin{array}{r}8.8 \\
-1.4 \\
2.3 \\
-6.2 \\
-2.4 \\
-0.6 \\
-0.5 \\
-0.1\end{array}$ & $<0.001$ \\
\hline $\begin{array}{l}\text { How many times do you } \\
\text { consume alcoholic } \\
\text { beverages in a week? }\end{array}$ & $\begin{array}{l}0.0 \\
1.0 \\
2.0 \\
3.0 \\
4.0 \\
5.0 \\
6.0 \\
7.0\end{array}$ & $\begin{array}{r}757 \\
586 \\
287 \\
170 \\
64 \\
59 \\
14 \\
84\end{array}$ & $\begin{array}{l}37.5 \\
29 \\
14.2 \\
8.4 \\
3.2 \\
2.9 \\
0.7 \\
4.2\end{array}$ & $\begin{array}{r}533 \\
376 \\
162 \\
94 \\
25 \\
33 \\
9 \\
44\end{array}$ & $\begin{array}{c}41.8 \\
29.5 \\
12.7 \\
7.4 \\
2 \\
2.6 \\
0.7 \\
3.4\end{array}$ & $\begin{array}{r}224 \\
210 \\
125 \\
76 \\
39 \\
26 \\
5 \\
40\end{array}$ & $\begin{array}{r}30.1 \\
28.2 \\
16.8 \\
10.2 \\
5.2 \\
3.5 \\
0.7 \\
5.4\end{array}$ & $\begin{array}{r}11.7 \\
1.3 \\
-4.1 \\
-2.8 \\
-3.3 \\
-0.1 \\
0.1 \\
-1.9\end{array}$ & $<0.001$ \\
\hline $\begin{array}{l}\text { Do you like salty (1) or } \\
\text { sweet (10)? }\end{array}$ & $\begin{array}{r}1.0 \\
2.0 \\
3.0 \\
4.0 \\
5.0 \\
6.0 \\
7.0 \\
8.0 \\
9.0 \\
10.0\end{array}$ & $\begin{array}{r}121 \\
63 \\
108 \\
134 \\
487 \\
121 \\
172 \\
329 \\
156 \\
330\end{array}$ & $\begin{array}{r}6.0 \\
3.1 \\
5.3 \\
6.6 \\
24.1 \\
6.0 \\
8.5 \\
16.3 \\
7.7 \\
16.3\end{array}$ & $\begin{array}{r}95 \\
44 \\
71 \\
84 \\
333 \\
70 \\
95 \\
180 \\
80 \\
224\end{array}$ & $\begin{array}{r}7.4 \\
3.4 \\
5.6 \\
6.6 \\
26.1 \\
5.5 \\
7.4 \\
14.1 \\
6.3 \\
17.5\end{array}$ & $\begin{array}{r}26 \\
19 \\
37 \\
50 \\
154 \\
51 \\
77 \\
149 \\
76 \\
106\end{array}$ & $\begin{array}{r}3.5 \\
2.5 \\
5.0 \\
6.7 \\
20.7 \\
6.8 \\
10.3 \\
20 \\
10.2 \\
14.2\end{array}$ & $\begin{array}{r}34 \\
1 \\
0.6 \\
-0.1 \\
5.4 \\
-1.3 \\
-2.9 \\
-5.9 \\
-3.9 \\
3.3\end{array}$ & $<0.001$ \\
\hline
\end{tabular}

of appetite, which influences central and peripheral signals implicated in the feedback controls of eating (Asarian and Geary, 2006).

In accordance with previous studies (Mattioli et al., 2013), women preferred more high-fibre foods, such as whole grain cereals (e.g. spelt and barley) and cooked vegetables, than men (Donkin et al., 1998). Consumption healthy foods, such as high-fibre foods, are more commonly attributed to the females. We discovered that men more often than women prefer eggs, meat and processed meat. This is probably because protein-rich foods, such as meat, represent strength and virility and are associated with men. In accordance previous studies, a higher proportion of men reported eating more meat, choosing fewer high-fibre foods, eating fewer low-fat foods and consuming more soft drinks than women (Shiferaw et al., 2012). As previously hypothesized, men probably make stronger associations between meat and healthiness than women do, and a "meat-strength/power association may mediate the meatmasculinity link readily observed across western cultures" (Love and Sulikowski, 2018). 


\begin{tabular}{|c|c|c|c|c|c|c|c|c|c|c|}
\hline \multirow[b]{2}{*}{ Question } & \multirow[b]{2}{*}{ Answers } & \multicolumn{2}{|c|}{$\begin{array}{c}\text { Total (2021 } \\
\text { subjects) }\end{array}$} & \multicolumn{2}{|c|}{$\begin{array}{l}\text { Women } \\
\text { (1276 } \\
\text { subjects) }\end{array}$} & \multicolumn{2}{|c|}{$\begin{array}{l}\text { Men (745 } \\
\text { subjects) }\end{array}$} & \multirow{2}{*}{$\begin{array}{c}\text { Difference } \\
{[\%]}\end{array}$} & \multirow[b]{2}{*}{$p$} & habits \\
\hline & & No. & {$[\%]$} & No. & {$[\%]$} & No. & {$[\%]$} & & & \\
\hline $\begin{array}{l}\text { How many times } \\
\text { do you eat a day? }\end{array}$ & $\begin{array}{l}1.0 \\
2.0 \\
3.0 \\
4.0 \\
5.0 \\
6.0 \\
7.0\end{array}$ & $\begin{array}{r}8 \\
112 \\
549 \\
603 \\
651 \\
80 \\
18\end{array}$ & $\begin{array}{r}0.4 \\
5.5 \\
27.2 \\
29.8 \\
32.2 \\
4.0 \\
0.9\end{array}$ & $\begin{array}{r}4 \\
54 \\
330 \\
386 \\
436 \\
55 \\
11\end{array}$ & $\begin{array}{r}0.3 \\
4.2 \\
25.9 \\
30.2 \\
34.2 \\
4.3 \\
0.9\end{array}$ & $\begin{array}{r}4 \\
58 \\
219 \\
217 \\
215 \\
25 \\
7\end{array}$ & $\begin{array}{r}0.5 \\
7.8 \\
29.4 \\
29.1 \\
28.9 \\
3.4 \\
0.9\end{array}$ & $\begin{array}{r}-0.2 \\
-3.5 \\
-3.5 \\
1.1 \\
5.3 \\
0.9 \\
-0.1\end{array}$ & $<0.001$ & \\
\hline $\begin{array}{l}\text { When are you } \\
\text { hungry during the } \\
\text { day? }\end{array}$ & $\begin{array}{l}\text { When I wake Up } \\
\text { During the morning } \\
\text { In The Afternoon } \\
\text { Before Dinner } \\
\text { After Dinner } \\
\text { I'm always hungry }\end{array}$ & $\begin{array}{r}145 \\
576 \\
418 \\
619 \\
188 \\
75\end{array}$ & $\begin{array}{r}7.2 \\
28.5 \\
20.7 \\
30.6 \\
9.3 \\
3.7\end{array}$ & $\begin{array}{r}104 \\
373 \\
276 \\
371 \\
107 \\
45\end{array}$ & $\begin{array}{r}8.1 \\
29.2 \\
21.6 \\
29.1 \\
8.4 \\
3.5\end{array}$ & $\begin{array}{r}41 \\
203 \\
142 \\
248 \\
81 \\
30\end{array}$ & $\begin{array}{r}5.5 \\
27.2 \\
19.1 \\
33.3 \\
10.9 \\
4\end{array}$ & $\begin{array}{l}2.6 \\
2 \\
2.6 \\
-4.2 \\
-2.5 \\
-0.5\end{array}$ & 0.024 & \\
\hline Do you eat fast? & $\begin{array}{l}\text { NO } \\
\text { YES }\end{array}$ & $\begin{array}{r}569 \\
1452\end{array}$ & $\begin{array}{l}28.1 \\
71.8\end{array}$ & $\begin{array}{l}415 \\
861\end{array}$ & $\begin{array}{l}32.5 \\
67.5\end{array}$ & $\begin{array}{l}154 \\
591\end{array}$ & $\begin{array}{l}20.7 \\
79.3\end{array}$ & $\begin{array}{r}11.8 \\
-11.8\end{array}$ & $<0.001$ & \\
\hline $\begin{array}{l}\text { Do you ever miss } \\
\text { meals? }\end{array}$ & $\begin{array}{l}\text { NO } \\
\text { YES (Total) } \\
\text { Yes. I have no time } \\
\text { Yes. for no reason } \\
\text { Yes. for craving before } \\
\text { meals } \\
\text { Yes. I always skip } \\
\text { breakfast }\end{array}$ & $\begin{array}{r}1263 \\
503 \\
41 \\
427 \\
35 \\
\\
\\
255\end{array}$ & $\begin{array}{c}62.5 \\
24.9 \\
2 \\
21.1 \\
1.7\end{array}$ & $\begin{array}{r}804 \\
344 \\
28 \\
288 \\
28\end{array}$ & $\begin{array}{r}63 \\
27 \\
2.2 \\
22.6 \\
2.2\end{array}$ & $\begin{array}{r}459 \\
159 \\
13 \\
139 \\
7\end{array}$ & $\begin{array}{r}61.6 \\
21.3 \\
1.7 \\
18.7 \\
0.9\end{array}$ & $\begin{array}{r}1.4 \\
5.6 \\
0.4 \\
3.9 \\
1.2 \\
-7\end{array}$ & $<0.001$ & \\
\hline $\begin{array}{l}\text { Do you happen to } \\
\text { eat uncontrollably } \\
\text { even if you're not } \\
\text { hungry? }\end{array}$ & $\begin{array}{l}\text { NO } \\
\text { YES (Total) } \\
\text { Yes. Every Day } \\
\text { Yes. Infrequent (1/month) } \\
\text { Yes. Often (>1/week) }\end{array}$ & $\begin{array}{r}478 \\
1543 \\
150 \\
683 \\
710\end{array}$ & $\begin{array}{r}23.6 \\
76.3 \\
7.4 \\
33.8 \\
35.1\end{array}$ & $\begin{array}{r}247 \\
1029 \\
101 \\
440 \\
488\end{array}$ & $\begin{array}{r}19.4 \\
80.6 \\
7.9 \\
34.5 \\
38.2\end{array}$ & $\begin{array}{r}231 \\
514 \\
49 \\
243 \\
222\end{array}$ & $\begin{array}{c}31 \\
69 \\
6.6 \\
32.6 \\
29.8\end{array}$ & $\begin{array}{r}-11.6 \\
11.6 \\
1.3 \\
1.9 \\
8.4\end{array}$ & $<0.001$ & \\
\hline $\begin{array}{l}\text { Do you eat out } \\
\text { with meals?" }\end{array}$ & $\begin{array}{l}\text { NO } \\
\text { YES (Total) } \\
\text { Often I eat at restaurant } \\
\text { in the evening } \\
\text { Refectory at lunch } \\
\text { At lunch I took foods from } \\
\text { home at work } \\
\text { Together at work in } \\
\text { restaurants }\end{array}$ & $\begin{array}{r}594 \\
1427 \\
149 \\
\\
265 \\
257 \\
\\
756\end{array}$ & $\begin{array}{r}29.4 \\
70.6 \\
7.4\end{array}$ & $\begin{array}{r}412 \\
864 \\
89 \\
\\
\\
130 \\
132 \\
\\
513\end{array}$ & $\begin{array}{c}32.2 \\
67.7 \\
7\end{array}$ & $\begin{array}{r}183 \\
562 \\
60\end{array}$ & $\begin{array}{r}24.5 \\
75.5 \\
8.1\end{array}$ & $\begin{array}{r}7.7 \\
-7.7 \\
-1.1 \\
\\
-7.9 \\
-6.3 \\
\\
7.6\end{array}$ & $<0.001$ & \\
\hline $\begin{array}{l}\text { Do you wake up to } \\
\text { eat at night? }\end{array}$ & $\begin{array}{l}\text { NO } \\
\text { YES (Total) } \\
\text { Every Day } \\
\text { Infrequent (1/month) } \\
\text { Often }(>1 / \text { week) }\end{array}$ & $\begin{array}{r}1558 \\
463 \\
45 \\
271 \\
147\end{array}$ & $\begin{array}{r}77.1 \\
22.9 \\
2.2 \\
13.4 \\
7.3\end{array}$ & $\begin{array}{r}1023 \\
253 \\
28 \\
146 \\
79\end{array}$ & $\begin{array}{r}80.2 \\
19.8 \\
2.2 \\
11.4 \\
6.2\end{array}$ & $\begin{array}{r}535 \\
210 \\
17 \\
125 \\
68\end{array}$ & $\begin{array}{r}71.8 \\
28.2 \\
2.3 \\
16.8 \\
9.1\end{array}$ & $\begin{array}{l}8.4 \\
-8.4 \\
3 \\
-1.8 \\
-2.9 \\
\quad(c o\end{array}$ & $\begin{array}{l}<0.001 \\
\text { ontinued) }\end{array}$ & $\begin{array}{r}\text { Table IV. } \\
\text { Relationship with } \\
\text { meals and sleep } \\
\text { habits between } \\
\text { women and men }\end{array}$ \\
\hline
\end{tabular}




\begin{tabular}{|c|c|c|c|c|c|c|c|c|c|}
\hline \multirow[b]{2}{*}{ Question } & \multirow[b]{2}{*}{ Answers } & \multicolumn{2}{|c|}{$\begin{array}{c}\text { Total (2021 } \\
\text { subjects) }\end{array}$} & \multicolumn{2}{|c|}{$\begin{array}{c}\text { Women } \\
(1276 \\
\text { subjects) }\end{array}$} & \multicolumn{2}{|c|}{$\begin{array}{l}\text { Men }(745 \\
\text { subjects) }\end{array}$} & \multirow{2}{*}{$\begin{array}{c}\text { Difference } \\
{[\%]}\end{array}$} & \multirow[b]{2}{*}{$p$} \\
\hline & & No. & {$[\%]$} & No. & {$[\%]$} & No. & {$[\%]$} & & \\
\hline \multirow{5}{*}{$\begin{array}{l}\text { Do you sleep well } \\
\text { at night? }\end{array}$} & NO & 1033 & 51.1 & 626 & 49.1 & 407 & 54.6 & -5.6 & $<0.001$ \\
\hline & I struggle to fall asleep & 238 & 11.8 & 138 & 10.8 & 100 & 13.4 & -2.6 & \\
\hline & $\begin{array}{l}\text { I wake up a lot sooner than } \\
\text { I would like }\end{array}$ & 180 & 8.9 & 108 & 8.5 & 72 & 9.7 & -1.2 & \\
\hline & $\begin{array}{l}\text { I wake up several times } \\
\text { during the night }\end{array}$ & 570 & 28.2 & 404 & 31.7 & 166 & 22.3 & 9.4 & \\
\hline & YES (Total) & 988 & 48.9 & 650 & 50.9 & 338 & 45.4 & 5.6 & \\
\hline
\end{tabular}

Table IV.

Men slept less, woke up more to eat at night and consequently skipped breakfast more often than women. Night eating syndrome has been positively associated with increased BMI, and the male gender and elevated psychological distress were associated with those who consumed nocturnal snacks (Colles et al., 2007). Men also ate out from home more often than women. In a large population-based cohort study, eating home cooked meals more frequently was associated with better dietary quality and lower adiposity (Mills et al., 2017). It was found that daily consumption of ready-made meals was associated with higher calorie intake and poor compliance with nutritional recommendations, and therefore could plausibly increase the risk of central obesity and fat deposition (Alkerwi et al., 2014).

\section{Limitations}

Our study has several limitations. Since it is a cross-sectional study, it cannot be used to analyse behaviour over a period, and it cannot distinguish cause and effect. Moreover, the proposed questionnaire has not been scientifically validated. Several likely relevant questions were not included, such as the participant's weight and profession, and for women the menstrual period, which as is known to have a large influence on the taste preferences of women.

\section{Conclusions}

The worldwide prevalence of obesity nearly tripled between 1975 and 2016 (Ulijaszek, 2003). The prevalence of obesity among men and women varies broadly, but in general more women are obese than men (Kanter and Caballero, 2012). Our findings revealed deep gender differences between men and women in terms of eating habits, the taste of food and the relationship with meals. This data suggests the need to create gender-specific programs to promote a healthy lifestyle, as previously demonstrated for individualized physical activity (Bellia et al., 2017). More research is needed to improve the understanding of these gender discrepancies and ultimately to improve lifestyle therapies.

Table shows differences between women and men in food preferences for whole grains foods, cereals, cooked vegetables, eggs, meat and processed meat. Chi-squared statistics were used to test the overall differences between male and female individuals (Statistical significance $p<0.001$, for eggs $p=0.03$ ).

The table shows the answers given by patients to questions about taste preferences. Only answers with statistically significant gender differences are reported (the others are present in the supplementary material). Chi-squared statistics were used to test the overall 
differences between male and female individuals (statistical significance set at $p \leq 0.05$ ). (Data as mean $\pm \mathrm{SD}$ ).

The table shows the answers given by patients to questions about food habits preferences. Only answers with statistically significant gender differences are reported (the others are present in the supplementary material). Chi-squared statistics were used to test the overall differences between male and female individuals (statistical significance set at $p \leq 0.05)$. (Data as mean $\pm \mathrm{SD}$ ).

The table shows the answers given by patients to questions about relationship with meals and sleep habits. Only answers with statistically significant gender differences are reported (the others are present in the supplementary material). Chi-squared statistics were used to test the overall differences between male and female individuals (statistical significance set at $p \leq 0.05$ ). (Data as mean $\pm \mathrm{SD}$ ).

AUTHORSHIP: Substantial contributions to conception and design, acquisition of data, or analysis and interpretation of data: ML Drafting the article or revising it critically for important intellectual content: ML, EP, GA, FI, MC, AB; final approval of the version to be published by ALL AUTHORS.

\section{References}

Alkerwi, A., Crichton, G. and Hébert, J. (2014), “Consumption of ready-made meals and increased risk of obesity: findings from the observation of cardiovascular risk factors in Luxembourg (ORISCAVLUX) study", British Journal of Nutrition, Vol. 113 No. 2, pp. 270-277.

Asarian, L. and Geary, N. (2006), "Modulation of appetite by gonadal steroid hormones", Philosophical Transactions of the Royal Society B: Biological Sciences, Vol. 361 No. 1471, pp. 1251-1263.

Baker, A. and Wardle, J. (2003), "Sex differences in fruit and vegetable intake in older adults", Appetite, Vol. 40 No. 3, pp. 269-275.

Beardsworth, A., Bryman, A., Keil, T., Goode, J., Haslam, C. and Lancashire, E. (2002), "Women, men and food: the significance of gender for nutritional attitudes and choices", British Food Journal, Vol. 104 No. 7, pp. 470-491.

Bellia, A., Iellamo, F., De Carli, E., Andreadi, A., Padua, E., Lombardo, M., Annino, G., Campoli, F., Tartaglione, S., D’Ottavio, S., Della-Morte, D. and Lauro, D. (2017), "Exercise individualized by TRIMPi method reduces arterial stiffness in early onset type 2 diabetic patients: a randomized controlled trial with aerobic interval training", International Journal of Cardiology, Vol. 248, pp. 314-319.

ICarbonneau, E., Bradette-Laplante, M., Lamarche, B., Provencher, V., Bégin, C., Robitaille, J., Desroches, S., Vohl, M.C., Corneau, L. and Lemieux, S. (2017), "Development and validation of the food liking questionnaire in a French-Canadian population", Nutrients, Vol. 9 No. 12, pp. 1337.

Colles, S., Dixon, J. and O'Brien, P. (2007), "Night eating syndrome and nocturnal snacking: association with obesity, binge eating and psychological distress", International Journal of Obesity, Vol. 31 No. 11, pp. 1722-1730.

Davy, S., Benes, B. and Driskell, J. (2006), "Sex differences in dieting trends, eating habits, and nutrition beliefs of a group of midwestern college students", Journal of the American Dietetic Association, Vol. 106 No. 10, pp. 1673-1677.

Di Cola, G., Jacoangeli, F., Jacoangeli, F., Lombardo, M. and Iellamo, F. (2014), "Cardiovascular disorders in anorexia nervosa and potential therapeutic targets", Internal and Emergency Medicine, Vol. 9 No. 7, pp. 717-721.

Djordjevic-Nikic, M. and Dopsaj, M. (2013), "Characteristics of eating habits and physical activity in relation to body mass index among adolescents", Journal of the American College of Nutrition, Vol. 32 No. 4, pp. 224-233. 
Donkin, A., Johnson, A., Lilley, J., Morgan, K., Neale, R., Page, R. and Silburn, R. (1998), "Gender and living alone as determinants of fruit and vegetable consumption among the elderly living at home in urban Nottingham", Appetite, Vol. 30 No. 1, pp. 39-51.

Franquesa, M., Pujol-Busquets, G., García-Fernández, E., Rico, L., Shamirian-Pulido, L., AguilarMartínez, A., Medina, F., et al. (2019), "Mediterranean diet and cardiodiabesity: a systematic review through evidence-based answers to key clinical questions", Nutrients, Vol. 11 No. 3, p. 655 .

Kanter, R. and Caballero, B. (2012), "Global gender disparities in obesity: a review", Advances in Nutrition, Vol. 3 No. 4, pp. 491-498.

Kiefer, I., Rathmanner, T. and Kunze, M. (2005), "Eating and dieting differences in men and women", The Journal of Men's Health and Gender, Vol. 2 No. 2, pp. 194-201.

Kubberød, E., Ueland, Ø., Tronstad, Å. and Risvik, E. (2002), "Attitudes towards meat and meateating among adolescents in Norway: a qualitative study", Appetite, Vol. 38 No. 1, pp. 53-62.

Lombardo, M., Magarotto, R., Padua, E., Caprio, M., Annino, G., Bellia, A. and Iellamo, F. (2017), "Ideal reduction of calories for greatest reduction of body fat and maintenance of lean body mass", The Journal of Aging Research and Clinical Practice, Vol. 1 No. 1.

Love, H. and Sulikowski, D. (2018), "Of meat and men: sex differences in implicit and explicit attitudes toward meat", Frontiers in Psychology, Vol. 9.

MacLean, P., Wing, R., Davidson, T., Epstein, L., Goodpaster, B., Hall, K., Levin, B., et al. (2014), "NIH working group report: innovative research to improve maintenance of weight loss", Obesity, Vol. 23 No. 1, pp. 7-15.

Manippa, V., Padulo, C., van der Laan, L. and Brancucci, A. (2017), "Gender differences in food choice: effects of superior temporal sulcus stimulation”, Frontiers in Human Neuroscience, Vol. 11.

Mattioli, A., Pennella, S., Pedrazzi, P., Rosi, C. and Farinetti, A. (2013), "Gender differences in adherence to mediterranean diet and risk of atrial fibrillation”, European Heart Journal, Vol. 34 No. suppl 1, pp. P4270-P4270.

Mills, S., Brown, H., Wrieden, W., White, M. and Adams, J. (2017), "Frequency of eating home cooked meals and potential benefits for diet and health: cross-sectional analysis of a population-based cohort study", International Journal of Behavioral Nutrition and Physical Activity, Vol. 14 No. 1.

$\mathrm{Nu}, \mathrm{C}$., MacLeod, P. and Barthelemy, J. (1996), "Effects of age and gender on adolescents' food habits and preferences", Food Quality and Preference, Vol. 7 Nos 3/4, pp. 251-262.

Padulo, C., Carlucci, L., Manippa, V., Marzoli, D., Saggino, A., Tommasi, L., Puglisi-Allegra, S., et al. (2017), "Valence, familiarity and arousal of different foods in relation to age, sex and weight", Food Quality and Preference, Vol. 57, pp. 104-113.

Shiferaw, B., Verrill, L., Booth, H., Zansky, S., Norton, D., Crim, S. and Henao, O. (2012), "SexBased differences in food consumption: foodborne diseases active surveillance network (FoodNet) population survey, 2006-2007”, Clinical Infectious Diseases, Vol. 54 No. suppl_5, pp. S453-S457.

Springmann, M., Clark, M., Mason-D’Croz, D., Wiebe, K., Bodirsky, B., Lassaletta, L., de Vries, W., et al. (2018), "Options for keeping the food system within environmental limits", Nature, Vol. 562 No. 7728 , pp. 519-525.

Ulijaszek, S. (2003), "Obesity: preventing and managing the global epidemic. Report of a WHO consultation. WHO technical report series 894. p. 252. (world health organization, Geneva, 2000.) SFr 56.00, ISBN 92-4-120894-5, paperback", Journal of Biosocial Science, Vol. 35 No. 4, pp. 624-625.

Wansink, B., Cheney, M. and Chan, N. (2003), "Exploring comfort food preferences across age and gender1”, Physiology Behavior, Vol. 79 Nos 4/5, pp. 739-747. 
Wardle, J., Haase, A., Steptoe, A., Nillapun, M., Jonwutiwes, K. and Bellisie, F. (2004), "Gender differences in food choice: the contribution of health beliefs and dieting", Annals of Behavioral Medicine, Vol. 27 No. 2, pp. 107-116.

Williams, R., Wood, L., Collins, C. and Callister, R. (2014), "Effectiveness of weight loss interventions - is there a difference between men and women: a systematic review", Obesity Reviews, Vol. 16 No. 2 , pp. 171-186.

Yumuk, V., Tsigos, C., Fried, M., Schindler, K., Busetto, L., Micic, D. and Toplak, H. (2015), "European guidelines for obesity management in adults", Obesity Facts, Vol. 8 No. 6, pp. 402-424.

\section{Corresponding author}

Mauro Lombardo can be contacted at: mauro.lombardo@unisanraffaele.gov.it

For instructions on how to order reprints of this article, please visit our website: 